Appl. Set-Valued Anal. Optim. 2 (2020), No. 3, pp. 273-283

Available online at http://asvao.biemdas.com

https://doi.org/10.23952/asvao.2.2020.3.02

\title{
ON SPLIT GENERALIZED MIXED EQUALITY EQUILIBRIUM AND SPLIT EQUALITY FIXED POINT PROBLEMS
}

\author{
CHARLES E. CHIDUME, ABUBAKAR ADAMU* \\ African University of Science and Technology, Abuja, Nigeria
}

\begin{abstract}
For $p \geq 2$, a new iterative algorithm is introduced and used to approximate a common element of the set of solutions of a split generalized mixed equality equilibrium problem and the set of solutions of a split equality fixed point problem for quasi- $\phi$-nonexpansive mappings in $p$-uniformly convex and uniformly smooth real Banach spaces. A strong convergence theorem is proved without any compactness-type assumption on the mappings. Furthermore, our theorem, which is applicable, in particular, in $L_{p}, l_{p}$ and the Sobolev spaces $W_{p}^{m}(\Omega)$ for $2 \leq p<\infty$, complements several important recent results that were established in 2-uniformly convex and uniformly smooth real Banach spaces.
\end{abstract}

Keywords. Fixed point; Quasi- $\phi$-nonexpansive; $p$-uniformly convex; Uniformly smooth.

\section{INTRODUCTION}

Let $C$ be a nonempty closed and convex subset of a real Banach space $E$ whose dual space is denoted by $E^{*}$. Let $\varphi: C \rightarrow \mathbb{R}$ be a mapping, $A: C \rightarrow E^{*}$ be a mapping and $f: C \times C \rightarrow \mathbb{R}$ be a bifunction. The generalized mixed equilibrium problem (GMEP) is a problem of finding

$$
u^{*} \in C \quad \text { such that } f\left(u^{*}, y\right)+\varphi(y)-\varphi\left(u^{*}\right)+\left\langle y-u^{*}, A u^{*}\right\rangle \geq 0, \forall y \in C .
$$

We denote the set of solutions of (1.1) by GMEP and it is given by

$$
\operatorname{GMEP}=\left\{u^{*} \in C: f\left(u^{*}, y\right)+\varphi(y)-\varphi\left(u^{*}\right)+\left\langle y-u^{*}, A u^{*}\right\rangle \geq 0, \forall y \in C\right\} .
$$

It is well known that this class of problems contains the class of equilibrium problems, optimization problems, fixed point problems, variational inequality problems, and so on. These classes of nonlinear problems have been studied extensively by various authors in the setting of real Hilbert spaces and more general real Banach spaces (see, e.g., [3, 9, 10, 11, 19, 21] and the references therein).

Let $H_{1}$ and $H_{2}$ be two real Hilbert spaces, and let $T: H_{1} \rightarrow H_{1}, S: H_{2} \rightarrow H_{2}$ be two nonlinear mappings with nonempty fixed point sets, $F(T):=\left\{x \in H_{1}: T x=x\right\}$ and $F(S):=\left\{u \in H_{2}: S u=\right.$ $u$ \}, respectively. Let $H_{3}$ be an arbitrary real Hilbert space. Let $A: H_{1} \rightarrow H_{3}$ and $B: H_{2} \rightarrow H_{3}$ be bounded linear mappings with adjoints $A^{*}$ and $B^{*}$, respectively. Moudafi [17] recently studied the following problem:

$$
\text { find } u^{*} \in F(T), \quad v^{*} \in F(S) \text { such that } A u^{*}=B v^{*} .
$$

\footnotetext{
*Corresponding author.
}

E-mail addresses: cchidume@ aust.edu.ng (C.E. Chidume), aadamu@aust.edu.ng (A. Adamu).

Received September 10, 2020; Accepted October 18, 2020. 
This problem, which is the so-called split equality fixed point problem (SEFPP), has recently attracted much attention and interest of lots of researchers due to its numerous applications in, for example, game theory, intensity modulated therapy treatment planning, decomposition methods for partial differential equations, fully discretized models of inverse problems which arise from phase retrievals and in medical image reconstructions (see, e.g., [1, 4, 7] and the references therein). We next denote the set of solutions of the split equality fixed point problem by SEFPP. If $H_{2}=H_{3}$ and $B$ is the identity mapping on $H_{2}$, the SEFPP is reduced to the so-called split common fixed point problem (SCFPP), introduced by Censor and Segal [8]. This problem has also been extensively studied by various authors (see, e.g., $[6,12,24]$ and the references therein).

In 2014, Bnouchachem [5] introduced the following split equilibrium problem in real Hilbert spaces. Let $f: C \times C \rightarrow \mathbb{R}$ and $g: Q \times Q \rightarrow \mathbb{R}$ be bifunctions, where $C$ and $Q$ are nonempty closed convex subsets of real Hilbert spaces, $H_{1}$ and $H_{2}$, respectively. Let $A: H_{1} \rightarrow H_{2}$ be a bounded linear mapping. The split equilibrium problem (SEQP) is as the following:

$$
\begin{gathered}
\text { find } u^{*} \in C \text { such that } f\left(u^{*}, y\right) \geq 0, \forall y \in C \text { and } \\
v=A u^{*} \in Q \text { solves } g(v, z) \geq 0, \forall z \in Q .
\end{gathered}
$$

In 2015, Zhao et al. [16] studied the following more general problem called the split equality equilibrium problem (SEEP), which is a problem of finding $\left(u^{*}, v^{*}\right) \in C \times Q$ such that

$$
f\left(u^{*}, y\right)+\vartheta(y)-\vartheta\left(u^{*}\right) \geq 0, \forall y \in C, g\left(v^{*}, z\right)+\varphi(z)-\varphi\left(v^{*}\right) \geq 0 \text { and } A u^{*}=B v^{*},
$$

where $f: C \times C \rightarrow \mathbb{R}$ and $g: Q \times Q \rightarrow \mathbb{R}$ are bifunctions, $\vartheta: C \rightarrow \mathbb{R} \cup\{\infty\}$ and $\varphi: Q \rightarrow$ $\mathbb{R} \cup\{\infty\}$ are proper lower semi-continuous functions, $A: C \subset H_{1} \rightarrow H_{3}$ and $B: Q \subset H_{2} \rightarrow H_{3}$ are bounded linear mappings, where $H_{1} H_{2}$ and $H_{3}$ are real Hilbert spaces. They proposed an iterative algorithm under their setting and proved that the sequence generated by their algorithm converges weakly to an element in $F(T) \cap F(S) \cap(S E F P P)$. Under additional assumption that $T$ and $S$ are semi-compact, they established the strong convergence of the sequence generated by their algorithm.

Very recently, Monday [18] studied the much more general problem, called the split generalized mixed equality equilibrium problem (SGMEEP) in real Banach spaces.

Setting 1.

(1) $E_{1}$ and $E_{2}$ are 2-uniformly convex and uniformly smooth real Banach spaces with dual spaces, $E_{1}^{*}$ and $E_{2}^{*}$, respectively. $E_{3}$ is an arbitrary smooth real Banach space.

(2) $C$ and $M$ are nonempty closed and convex subsets of $E_{1}$ and $E_{2}$, respectively. $f: C \times C$ and $g: M \times M \rightarrow \mathbb{R}$ are bifunctionals.

(3) $\vartheta: C \rightarrow \mathbb{R} \cup\{\infty\}$ and $\varphi: M \rightarrow \mathbb{R} \cup\{\infty\}$ are proper lower semi-continous and convex functions.

(4) $U: C \rightarrow E_{1}^{*}$ and $V: M \rightarrow E_{2}^{*}$ are continuous monotone mappings.

(5) $A: E_{1} \rightarrow E_{3}$ and $B: E_{2} \rightarrow E_{3}$ are bounded linear mappings.

The SGMEEP is a problem of finding $\left(u^{*}, v^{*}\right) \in C \times M$ such that

$$
\begin{aligned}
f\left(u^{*}, y\right)+\psi(y)-\psi\left(u^{*}\right)+\left\langle y-u^{*}, U u^{*}\right\rangle & \geq 0, \forall y \in C, \\
g\left(v^{*}, z\right)+\varphi(z)-\varphi\left(v^{*}\right)+\left\langle z-v^{*}, V v^{*}\right\rangle & \geq 0, \forall z \in M, \text { and } A u^{*}=B v^{*} .
\end{aligned}
$$

Monday [18] studied the SGMEEP in conjunction with the SEFPP for quasi- $\phi$-nonexpansive mappings under the above setting. He constructed an iterative algorithm and proved that the 
sequence generated by his algorithm converges strongly to an element in the intersection of the solution set of the SGMEEP and the solution set of the SEFPP. The quasi- $\phi$-nonexpansive operators were not assumed to be semi-compact. Monday's theorem improves and complements several important recent results.

Remark 1.1. 2-uniformly convex real Banach spaces are more general than Hilbert spaces (for example, they include $L_{p}, l_{p}$ and the Sobolev spaces $W_{p}^{m}(\Omega)$, for $1<p \leq 2$ ), while $L_{p}, l_{p}$ and the Sobolev spaces $W_{p}^{m}(\Omega)$, for $2<p<\infty$ are not 2-uniformly convex.

It is our purpose in this paper to study the SGMEEP and the SEFPP for quasi- $\phi$-nonexpansive mappings in $p$-uniformly convex and uniformly smooth real Banach spaces, $2 \leq p<\infty$. We propose a new iterative algorithm under our setting and prove that the sequence generated by our algorithm converges strongly to an element in the intersection of the solution set of SGMEEP and the solution set of the SEFPP. Moreover, our operators are not assumed to be semi-compact. Our theorem is, in particular, applicable in $L_{p}, l_{p}$ and the Sobolev spaces $W_{p}^{m}(\Omega)$, for all $p$ such that $2 \leq p<\infty$.

\section{PRELIMINARIES}

Let $E$ be a strictly convex and smooth real Banach space. For $p>1$, define $J_{p}: E \rightarrow 2^{E^{*}}$ by

$$
J_{p}(x):=\left\{u^{*} \in E^{*}:\left\langle x, u^{*}\right\rangle=\|x\|\left\|u^{*}\right\|,\left\|u^{*}\right\|=\|x\|^{p-1}\right\} .
$$

$J_{p}$ is called the generalized duality mapping on $E$. If $p=2, J_{2}$ is called the normalized duality map and is denoted by $J$. In a real Hilbert space $H, J$ is the identity map on $H$. It is easy to see from the definition that

$$
J_{p}(x)=\|x\|^{p-2} J x, \quad \text { and } \quad\left\langle x, J_{p} x\right\rangle=\|x\|^{p}, \forall x \in E .
$$

It is well-known that if $E$ is smooth, then $J$ is single-valued and if $E$ is strictly convex, $J$ is one-to-one, and $J$ is surjective if $E$ is reflexive.

Let $E$ be a reflexive, strictly convex and smooth real Banach space with dual space $E^{*}$. For $p>1$, Chidume [13] defined the following functionals: $\phi_{p}: E \times E \rightarrow \mathbb{R}^{+}$by

$$
\phi_{p}(x, y):=\|x\|^{p}-p\left\langle x, J_{p} y\right\rangle+\left\|J_{p} y\right\|^{p}, \forall x, y \in E,
$$

and $V_{p}: E \times E^{*} \rightarrow \mathbb{R}^{+}$by

$$
V_{p}\left(x, x^{*}\right):=\|x\|^{p}-p\left\langle x, x^{*}\right\rangle+\left\|x^{*}\right\|^{p}, \forall x \in E, x^{*} \in E^{*} .
$$

It is clear from these definitions that

$$
V_{p}\left(x, x^{*}\right)=\phi_{p}\left(x, J_{p}^{-1} x^{*}\right), \forall x \in E, x^{*} \in E^{*} .
$$

Remark 2.1. If $p=2$, we denote $\phi_{2}(x, y)$ simply as $\phi(x, y)$. So,

$$
\phi(x, y)=\|x\|^{2}-2\langle x, J y\rangle+\|y\|^{2}, \forall x, y \in E .
$$

Definition 2.1. Let $C$ be a nonempty subset of a real normed space $E$ and let $T: C \rightarrow C$ be a mapping. Then,

(i) $T$ is called quasi- $\phi$-nonexpansive [20] if $F(T):=\{x \in C: T x=x\} \neq \emptyset$, and

$$
\phi_{p}\left(x^{*}, T x\right) \leq \phi_{p}\left(x^{*}, x\right), \forall x \in C, x^{*} \in F(T) .
$$

(ii) $T$ is said to be closed if for any sequence $\left\{x_{n}\right\} \subset C$ and $x_{n} \rightarrow x$ and $T x_{n} \rightarrow y$, then $y=T x$. 
In the sequel, we shall need the following lemmas, which was recently established by Chidume [13].

Lemma 2.1. Let $E$ be a reflexive, strictly convex and smooth real Banach space. Then, for $p>1$

$$
V_{p}\left(u, u^{*}\right)+p\left\langle J_{p}^{-1} u^{*}-u, v^{*}\right\rangle \leq V_{p}\left(u, u^{*}+v^{*}\right), \forall u \in E, u^{*}, v^{*} \in E^{*} .
$$

Lemma 2.2. For $p>1$, let $E$ be a p-uniformly convex and smooth real Banach space. Let $D$ be a nonempty closed and convex subset of $E$. Let $x_{1} \in E$ and $P_{D}: E \rightarrow D$ be the metric projection of $E$ onto D. Then

$$
x^{*}=P_{D} x_{1}, \Leftrightarrow\left\langle x^{*}-z, J_{p}\left(x_{1}-x^{*}\right)\right\rangle \geq 0, \forall z \in D .
$$

Lemma 2.3. Let $E$ be a p-uniformly convex and smooth real Banach space with dual space $E^{*}$. For $p>1$, let $J_{p}: E \rightarrow E^{*}$ be the generalized duality map. Then,

$$
\left\|J_{p}^{-1} x-J_{p}^{-1} y\right\| \leq \kappa_{p}\|x-y\|^{\frac{1}{p-1}}, \forall x, y \in E
$$

where $\kappa_{p}=\left(\frac{1}{c_{2}}\right)^{\frac{1}{p-1}}$ for some constant $c_{2}>0$.

Lemma 2.4. Let $E$ be a reflexive, strictly convex and smooth real Banach space. Then, for $p>1$, there exists a constant $c_{p}>0$ such that, for all $x, u, v \in E$,

$$
\phi_{p}\left(x, J_{p}^{-1}\left(\lambda J_{p} u+(1-\lambda) J_{p} v\right)\right) \leq \lambda \phi_{p}(x, u)+(1-\lambda) \phi_{p}(x, v)-c_{p} w_{p}(\lambda)\left\|J_{p} u-J_{p} v\right\|^{p},
$$

where $w_{p}(\lambda)=\lambda^{p}(1-\lambda)+\lambda(1-\lambda)^{p}$.

We shall also need the following well known lemmas.

Lemma 2.5. [2] Let D be a nonempty closed and convex subset of a reflexive, strictly convex and smooth real Banach space E. Then,

$$
\phi\left(u, \Pi_{D} y\right)+\phi\left(\Pi_{D} y, y\right) \leq \phi(u, y), \forall u \in D, y \in E,
$$

where $\Pi_{D}$ is the generalized projection of $E$ onto $D$.

Lemma 2.6. [14] Let $E$ be a uniformly convex and uniformly smooth real Banach space and let $\left\{x_{n}\right\}$ and $\left\{y_{n}\right\}$ be two sequences of E. If $\phi\left(x_{n}, y_{n}\right) \rightarrow 0$ and either $\left\{x_{n}\right\}$ or $\left\{y_{n}\right\}$ is bounded, then $\left\|x_{n}-y_{n}\right\| \rightarrow 0$, as $n \rightarrow \infty$.

Lemma 2.7. [23] For $p>1$, let E be a p-uniformly convex real Banach space. Then, there exists a constant $c_{p}>0$ such that for all $x, y \in E$, the following inequality holds:

$$
\|\alpha x+(1-\alpha) y\|^{p} \leq \alpha\|x\|^{p}+(1-\alpha)\|y\|^{p}-c_{p} w_{p}(\alpha)\|x-y\|^{p} .
$$

2.1. Analytical representations of generalized duality mappings in $L_{p}, l_{p}$, and $W_{m}^{p}$, spaces, $1<p<\infty$. Using the analytic representation of the normalized duality mappings in $L_{p}, l_{p}$, and $W_{m}^{p}, 1<p<\infty$ (see, e.g., Lindenstrauss and Tzafriri [15]) and the relation $J_{p}(x)=\|x\|^{p-2} J(x)$, we obtain the analytical representations of generalized duality mappings in these spaces as follows:

$$
\begin{gathered}
J_{p} z=y \in l_{q}, y=\left\{\left|z_{1}\right|^{p-2} z_{1},\left|z_{2}\right|^{p-2} z_{2}, \ldots\right\}, z=\left\{z_{1}, z_{2}, \ldots\right\}, \\
J_{p}^{-1} z=y \in l_{p}, y=\left\{\left|z_{1}\right|^{q-2} z_{1},\left|z_{2}\right|^{q-2} z_{2}, \ldots\right\}, z=\left\{z_{1}, z_{2}, \ldots\right\}, \\
J_{p} z=|z(s)|^{p-2} z(s) \in L_{q}(G), s \in G,
\end{gathered}
$$




$$
J_{p}^{-1} z=|z(s)|^{q-2} z(s) \in L_{p}(G), s \in G,
$$

and

$$
J_{p} z=\sum_{|\alpha| \leq m}(-1)^{|\alpha|} D^{\alpha}\left(\left|D^{\alpha} z(s)\right|^{p-2} D^{\alpha} z(s)\right) \in W_{-m}^{q}(G), \quad m>0, s \in G
$$

\section{MAin RESUlTS}

We are now ready to prove our main theorem. Before we go, we need to prove the following new lemma, which will play a key role in the proof of our theorem and which is also of independent interest.

Lemma 3.1. Let $E$ be a reflexive, strictly convex and smooth real Banach space and let $C$ be a nonempty closed and convex subset of E. Let $r>0, p>1$ and $x \in E$. Define a mapping $T_{r}: E \rightarrow C$ by

$$
T_{r}(x):=\left\{z \in C: \Theta(z, y)+\frac{1}{r}\left\langle y-z, J_{p} z-J_{p} x\right\rangle \geq 0, \forall y \in C\right\},
$$

where $\Theta$ is any bifunction. Then, $T_{r}$ is single valued. For $p \geq 2$, the following inequality holds:

$$
\phi_{p}\left(q, T_{r}(x)\right)+\phi_{p}\left(T_{r}(x), x\right) \leq \phi_{p}(q, x), \forall q \in F\left(T_{r}\right) .
$$

Proof. From [22], we have that $T_{r}$ is single valued. Using the definition of $\phi_{p}$ and the definition of $T_{r}$, we have

$$
\begin{aligned}
\phi_{p}\left(q, T_{r}(x)\right)+\phi_{p}\left(T_{r}(x), x\right)= & \|q\|^{p}-p\left\langle q, J_{p} T_{r}(x)\right\rangle+\left\|J_{p} T_{r} x\right\|^{p}+\left\|T_{r}(x)\right\|^{p} \\
& -p\left\langle T_{r}(x), J_{p} x\right\rangle+\left\|J_{p} x\right\|^{p} \\
= & \|q\|^{p}-p\left\langle q, J_{p} T_{r}(x)\right\rangle+\left\|J_{p} T_{r} x\right\|^{p}+\left\|T_{r}(x)\right\|^{p}-p\left\langle q, J_{p} x\right\rangle \\
& -p\left\langle T_{r}(x)-q, J_{p} x\right\rangle+\left\|J_{p} x\right\|^{p} \\
= & \phi_{p}(q, x)-p\left\langle q, J_{p} T_{r}(x)\right\rangle+2\left\langle T_{r}(x), J_{p} T_{r}(x)\right\rangle \\
& -p\left\langle T_{r}(x)-q, J_{p} x\right\rangle \\
\leq & \phi_{p}(q, x)-p\left\langle q-T_{r}(x), J_{p} T_{r}(x)\right\rangle+p\left\langle q-T_{r}(x), J_{p} x\right\rangle \\
= & \phi_{p}(q, x)-p\left\langle q-T_{r}(x), J_{p} T_{r}(x)-J_{p} x\right\rangle \\
\leq & \phi_{p}(q, x) .
\end{aligned}
$$

This completes the proof.

Basic assumption. Let $C$ be a nonempty closed and convex subset of a real Banach space $E$ with dual space $E^{*}$. Let $g: C \rightarrow \mathbb{R}$ be a lower semi-continuous and convex function, and let $A$ : $C \rightarrow E^{*}$ be continuous and monotone. For solving the generalized mixed equality equilibrium problem, we assume that each bifunction $f: C \times C \rightarrow \mathbb{R}$ satisfies the following conditions:

(A1) $f(u, u)=0, \forall u \in C$;

(A2) $f$ is monotone, i.e., $f(u, v)+f(v, u)=0, \forall u, v \in C$;

(A3) $\limsup f(u+t(z-u), v) \leq f(u, v), \forall u, v, z \in C$;

$$
t \downarrow 0
$$

(A4) $f(u, \cdot)$ is convex and lower semi-continous, $\forall u \in C$.

For Theorem 3.1 below, we have the following setting. Setting 2. 
(1) For $p \geq 2, E_{1}$ and $E_{2}$ are $p$-uniformly convex and uniformly smooth real Banach spaces with dual spaces, $E_{1}^{*}$ and $E_{2}^{*}$, respectively. $E_{3}$ is an arbitrary smooth real Banach space with dual space, $E_{3}^{*}$.

(2) $C$ and $M$ are nonempty closed and convex subsets of $E_{1}$ and $E_{2}$, respectively.

(3) $\varphi: C \rightarrow \mathbb{R} \cup\{\infty\}$ and $\vartheta: M \rightarrow \mathbb{R} \cup\{\infty\}$ are proper lower semi-continuous functions.

(4) $f: C \times C \rightarrow \mathbb{R}$ and $g: M \times M \rightarrow \mathbb{R}$ are bifunctionals satisfying (A1)-(A4).

(5) $U: C \rightarrow E^{*}$ and $V: M \rightarrow E_{2}^{*}$ are continuous and monotone mappings.

(6) $T: E_{1} \rightarrow E_{1}$ and $S: E_{2} \rightarrow E_{2}$ are closed quasi- $\phi$-nonexpansive mappings.

(7) $A: E_{1} \rightarrow E_{3}$ and $B: E_{2} \rightarrow E_{3}$ are bounded linear mappings with adjoints $A^{*}$ and $B^{*}$, respectively.

(8) $\alpha \in(0,1)$ and $\mu$ is such that $0<\mu<\left[\frac{1}{\kappa_{p}\left(\|A\|^{\frac{p}{p-1}}+\|B\|^{\frac{p}{p-1}}\right)}\right]^{p-1}, p \geq 2$.

(9) $J_{p E_{i}}$ denotes the generalized duality map on $E_{i}, i=1,2,3$, respectively.

\section{Algorithm 3.1.}

$$
\left\{\begin{array}{l}
x_{1} \in E_{1}, y_{1} \in E_{2}, C_{1}=E_{1}, Q_{1}=E_{2}, \\
u_{n}=T_{r} x_{n}, v_{n}=T_{r} y_{n}, e_{n}=J_{p E_{3}}\left(A u_{n}-B v_{n}\right), \\
\theta_{n}=J_{p E_{1}}^{-1}\left(J_{p E_{1}} u_{n}-\mu A^{*} e_{n}\right), \delta_{n}=J_{p E_{2}}^{-1}\left(J_{p E_{2}} v_{n}+\mu B^{*} e_{n}\right), \\
z_{n}=J_{p E_{1}}^{-1}\left(\alpha J_{p E_{1}} x_{n}+(1-\alpha) J_{p E_{1}} T \theta_{n}\right), \\
w_{n}=J_{p E_{2}}^{-1}\left(\alpha J_{p E_{2}} y_{n}+(1-\alpha) J_{p E_{2}} S \delta_{n}\right), \\
C_{n+1}=\left\{c \in C_{n}: \phi_{p}\left(c, z_{n}\right) \leq \phi_{p}\left(c, x_{n}\right)\right\}, \\
Q_{n+1}=\left\{q \in Q_{n}: \phi_{p}\left(q, w_{n}\right) \leq \phi_{p}\left(q, y_{n}\right)\right\}, \\
x_{n+1}=\Pi_{C_{n+1}} x_{1}, \quad y_{n+1}=\Pi_{Q_{n+1}} y_{1}, \forall n \geq 1,
\end{array}\right.
$$

where $\Pi$ denotes the generalized projection of Alber [2].

Theorem 3.1. Let $\left\{\left(x_{n}, y_{n}\right)\right\} \subset E_{1} \times E_{2}$ be a sequence generated by Algorithm 3.1. Assume $\Omega:=S G M E E P \cap S E F P P \neq \emptyset$. Then, $\left\{\left(x_{n}, y_{n}\right)\right\}$ converges strongly to a point $\left(x^{*}, y^{*}\right) \in \Omega$.

Proof. We divide the proof into four steps.

Step 1. Show that the sequences $\left\{x_{n}\right\}$ and $\left\{y_{n}\right\}$ are well defined.

First, we show that $C_{n}$ and $Q_{n}$ are closed and convex. Clearly, $C_{1}=E_{1}$ and $Q_{1}=E_{2}$ are closed and convex. Assume $C_{n}$ and $Q_{n}$ are closed and convex for some $n \geq 1$. From the definition of $C_{n+1}$, we obtain that

$$
C_{n+1}=\left\{c \in C_{n}: p\left\langle c, J_{p E_{1}} x_{n}-J_{p E_{1}} z_{n}\right\rangle \leq\left\|J_{p} x_{n}\right\|^{p}-\left\|J_{p} z_{n}\right\|^{p}\right\} .
$$

Thus, $C_{n+1}$ is closed and convex. Similarly, $Q_{n+1}$ is closed and convex. Hence, $C_{n}$ and $Q_{n}$ are closed and convex, for all $n \geq 1$.

Note that $\Omega \subset C_{1} \times Q_{1}$. Assume that $\Omega \subset C_{n} \times Q_{n}$ for some integer $n \geq 1$. Let $(c, q) \in \Omega$. Then, by Lemma 2.4 and the fact that $T$ is quasi- $\phi$-nonexpansive, we have

$$
\begin{aligned}
\phi_{p}\left(c, z_{n}\right) & =\phi_{p}\left(c, J_{p E_{1}}^{-1}\left(\alpha J_{p E_{1}} x_{n}+(1-\alpha) J_{p E_{1}} T \theta_{n}\right)\right) \\
& \leq \alpha \phi_{p}\left(c, x_{n}\right)+(1-\alpha) \phi_{p}\left(c, T \theta_{n}\right)-c_{p} w_{p}(\alpha)\left\|J_{p E_{1}} x_{n}-J_{p E_{1}} T \theta_{n}\right\|^{p} \\
& \leq \alpha \phi_{p}\left(c, x_{n}\right)+(1-\alpha) \phi_{p}\left(c, \theta_{n}\right)-c_{p} w_{p}(\alpha)\left\|J_{p E_{1}} x_{n}-J_{p E_{1}} T \theta_{n}\right\|^{p} .
\end{aligned}
$$


Now, using definition of $V_{p}$, Lemmas 2.1 and 3.1, we have

$$
\begin{aligned}
\phi_{p}\left(c, \theta_{n}\right) & =\phi_{p}\left(c, J_{p E_{1}}^{-1}\left(J_{p E_{1}} u_{n}-\mu A^{*} e_{n}\right)\right) \\
& =V_{p}\left(c, J_{p E_{1}} u_{n}-\mu A^{*} e_{n}\right) \\
& \leq V_{p}\left(c, J_{p E_{1}} u_{n}\right)-p \mu\left\langle J_{p E_{1}}^{-1}\left(J_{p E_{1}} u_{n}-\mu A^{*} e_{n}\right)-c, A^{*} e_{n}\right\rangle \\
& =\phi_{p}\left(c, u_{n}\right)-p \mu\left\langle\theta_{n}-c, A^{*} e_{n}\right\rangle \\
& =\phi_{p}\left(c, u_{n}\right)-p \mu\left\langle A\left(\theta_{n}-c\right), e_{n}\right\rangle \\
& \leq \phi_{p}\left(c, x_{n}\right)-p \mu\left\langle A\left(\theta_{n}-c\right), e_{n}\right\rangle .
\end{aligned}
$$

Substituting this inequality into inequality (3.2), we obtain

$$
\phi_{p}\left(c, z_{n}\right) \leq \phi_{p}\left(c, x_{n}\right)-p \mu(1-\alpha)\left\langle A\left(\theta_{n}-c\right), e_{n}\right\rangle-c_{p} w_{p}(\alpha)\left\|J_{p E_{1}} x_{n}-J_{p E_{1}} T \theta_{n}\right\|^{p} .
$$

Similarly, we obtain that

$$
\phi_{p}\left(q, w_{n}\right) \leq \phi_{p}\left(q, y_{n}\right)-p \mu(1-\alpha)\left\langle B\left(q-\delta_{n}\right), e_{n}\right\rangle-c_{p} w_{p}(\alpha)\left\|J_{p E_{2}} y_{n}-J_{p E_{2}} S \delta_{n}\right\|^{p} .
$$

Adding inequalities (3.4) and (3.5) and using the fact that $A c=B q$, we have

$$
\begin{aligned}
\phi_{p}\left(c, z_{n}\right)+\phi_{p}\left(q, w_{n}\right) \leq & \phi_{p}\left(c, x_{n}\right)+\phi_{p}\left(q, y_{n}\right)-p \mu(1-\alpha)\left\langle A \theta_{n}-B \delta_{n}, e_{n}\right\rangle \\
& -c_{p} w_{p}(\alpha)\left[\left\|J_{p E_{1}} x_{n}-J_{p E_{1}} T \theta_{n}\right\|^{p}+\left\|J_{p E_{2}} y_{n}-J_{p E_{2}} S \delta_{n}\right\|^{p}\right] .
\end{aligned}
$$

Using this inequality and Lemma 2.3, we arrive at

$$
\begin{aligned}
-(1-\alpha) p \mu\left\langle A \theta_{n}-B \delta_{n}, e_{n}\right\rangle= & -(1-\alpha) p \mu\left[\left\langle A u_{n}-B v_{n}, e_{n}\right\rangle-\left\langle A u_{n}-A \theta_{n}, e_{n}\right\rangle\right. \\
& \left.-\left\langle B \delta_{n}-B v_{n}, e_{n}\right\rangle\right] \\
\leq & -(1-\alpha) p \mu\left\|A u_{n}-B v_{n}\right\|^{p}+(1-\alpha) p \mu\left[\|A\|\left\|u_{n}-\theta_{n}\right\|\left\|e_{n}\right\|\right. \\
& \left.+\|B\|\left\|\delta_{n}-v_{n}\right\|\left\|e_{n}\right\|\right] .
\end{aligned}
$$

But

$$
\begin{aligned}
\left\|\theta_{n}-u_{n}\right\| & =\left\|J_{p E_{1}}^{-1}\left(J_{p E_{1}} u_{n}-\mu A^{*} e_{n}\right)-J_{p E_{1}}^{-1} J_{p E_{1}} u_{n}\right\| \\
& \leq \kappa_{p}\left\|\mu A^{*} e_{n}\right\|^{\frac{1}{p-1}} \leq \kappa_{p} \mu^{\frac{1}{p-1}}\|A\|^{\frac{1}{p-1}}\left\|e_{n}\right\|^{\frac{1}{p-1}}
\end{aligned}
$$

and

$$
\begin{aligned}
\left\|\delta_{n}-v_{n}\right\| & =\left\|J_{p E_{2}}^{-1}\left(J_{p E_{2}} v_{n}+\mu B^{*} e_{n}\right)-J_{p E_{1}}^{-1} J_{p E_{1}} v_{n}\right\| \\
& \leq \kappa_{p}\left\|\mu B^{*} e_{n}\right\|^{\frac{1}{p-1}} \leq \kappa_{p} \mu^{\frac{1}{p-1}}\|B\|^{\frac{1}{p-1}}\left\|e_{n}\right\|^{\frac{1}{p-1}}
\end{aligned}
$$

Substituting (3.8) and (3.9) into (3.7), we obtain

$$
\begin{gathered}
-(1-\alpha) \mu\left\langle A \theta_{n}-B \delta_{n}, e_{n}\right\rangle \leq- \\
\left.+\|B\|^{\frac{p}{p-1}}\right]\left\|e_{n}\right\|^{\frac{p}{p-1}} \\
=-(1-\alpha) p \mu\left[1-\mu^{\frac{1}{p-1}} \kappa_{p}\left(\|A\|^{\frac{p}{p-1}}+\|B\|^{\frac{p}{p-1}}\right)\right] \times \\
\left\|A u_{n}-B v_{n}\right\|^{p} \leq 0,
\end{gathered}
$$

due to

$$
0<\mu<\left[\frac{1}{\kappa_{p}\left(\|A\|^{\frac{p}{p-1}}+\|B\|^{\frac{p}{p-1}}\right)}\right]^{p-1} .
$$


Let $\zeta=(1-\alpha) p \mu\left[1-\mu^{\frac{1}{p-1}} \kappa_{p}\left(\|A\|^{\frac{p}{p-1}}+\|B\|^{\frac{p}{p-1}}\right)\right]$. Then, from inequality (3.6), and using inequality (3.10), we obtain that

$$
\begin{aligned}
\phi_{p}\left(c, z_{n}\right)+\phi_{p}\left(q, w_{n}\right) \leq & \phi_{p}\left(c, x_{n}\right)+\phi_{p}\left(q, y_{n}\right)-\zeta\left\|A u_{n}-B v_{n}\right\|^{p} \\
& -c_{p} w_{p}(\alpha)\left[\left\|J_{p E_{1}} x_{n}-J_{p E_{1}} T \theta_{n}\right\|^{p}+\left\|J_{p E_{2}} y_{n}-J_{p E_{2}} S \delta_{n}\right\|\right] \\
\leq & \phi_{p}\left(c, x_{n}\right)+\phi_{p}\left(q, y_{n}\right) .
\end{aligned}
$$

This implies that $(c, q) \in C_{n+1} \times Q_{n+1}$. Hence, $\Omega \subset C_{n} \times Q_{n}, \forall n \geq 1$. These conclusions imply that $C_{n}$ and $Q_{n}$ are nonempty closed and convex, for all $n \geq 1$. Therefore, $\left\{x_{n}\right\}$ and $\left\{y_{n}\right\}$ are well defined.

Step 2. Prove that the sequences $\left\{x_{n}\right\}$ and $\left\{y_{n}\right\}$ are convergent.

This is standard by means of Lemmas 2.6 and 2.7. It follows exactly as in [18] that $x_{n} \rightarrow x^{*}$ and $y_{n} \rightarrow y^{*}$, as $n \rightarrow \infty$, for some $x^{*} \in E_{1}$ and $y^{*} \in E_{2}$. Furthermore, $z_{n} \rightarrow x^{*}$ and $w_{n} \rightarrow y^{*}$, as $n \rightarrow \infty$.

Step 3. Show that $\lim _{n \rightarrow \infty}\left\|u_{n}-x_{n}\right\|=0$ and $\lim _{n \rightarrow \infty}\left\|v_{n}-y_{n}\right\|=0$.

From inequality (3.11), we have

$$
\begin{gathered}
c_{p} w_{p}(\alpha)\left[\left\|J_{p E_{1}} x_{n}-J_{p E_{1}} T \theta_{n}\right\|^{p}+\left\|J_{p E_{2}} y_{n}-J_{p E_{2}} S \delta_{n}\right\|^{p}\right]+\zeta\left\|A u_{n}-B v_{n}\right\| \\
\leq \phi_{p}\left(c, x_{n}\right)-\phi_{p}\left(c, z_{n}\right)+\phi_{p}\left(q, y_{n}\right)-\phi_{p}\left(q, w_{n}\right) .
\end{gathered}
$$

This implies that

$$
\begin{gathered}
\lim _{n \rightarrow \infty}\left\|J_{p E_{1}} x_{n}-J_{p E_{1}} T \theta_{n}\right\|=0, \quad \lim _{n \rightarrow \infty}\left\|J_{p E_{2}} y_{n}-J_{p E_{2}} S \delta_{n}\right\|=0, \\
\text { and } \lim _{n \rightarrow \infty}\left\|A u_{n}-B v_{n}\right\|=0 .
\end{gathered}
$$

Furthermore, using the uniform continuity of $J_{p}^{-1}$ on bounded sets, we have

$$
\lim _{n \rightarrow \infty}\left\|x_{n}-T \theta_{n}\right\|=0 \quad \text { and } \quad \lim _{n \rightarrow \infty}\left\|y_{n}-S \delta_{n}\right\|=0 .
$$

Using Lemma 2.3 and equation (3.13) we have that

$$
\begin{aligned}
\left\|u_{n}-\theta_{n}\right\| & \leq \kappa_{p}\left\|\mu A^{*} e_{n}\right\|^{\frac{1}{p-1}} \\
& \leq \kappa_{p} \mu^{\frac{1}{p-1}}\|A\|^{\frac{1}{p-1}}\left\|A u_{n}-B v_{n}\right\|,
\end{aligned}
$$

which implies that $\lim _{n \rightarrow \infty}\left\|u_{n}-\theta_{n}\right\|=0$. Similarly, $\lim _{n \rightarrow \infty}\left\|v_{n}-\delta_{n}\right\|=0$. Also, using equations (3.13) and Lemma 2.3, we have

$$
\left\|z_{n}-x_{n}\right\| \leq \kappa_{p}(1-\alpha)^{\frac{1}{p-1}}\left\|J_{p E_{1}} T \theta_{n}-J_{p E_{1}} x_{n}\right\|^{\frac{1}{p-1}} \rightarrow 0, \text { as } n \rightarrow \infty .
$$

Thus, $\lim _{n \rightarrow \infty}\left\|z_{n}-x_{n}\right\|=0$. Similarly, we have

$$
\lim _{n \rightarrow \infty}\left\|w_{n}-y_{n}\right\|=0 \text {. }
$$

Next, we show that $\lim _{n \rightarrow \infty} \phi_{p}\left(u_{n}, x_{n}\right)=0$ and $\lim _{n \rightarrow \infty} \phi_{p}\left(v_{n}, y_{n}\right)=0$. By Lemma 3.1, we have that

$$
\text { and } \quad \begin{array}{ll}
\phi_{p}\left(c, u_{n}\right) \leq \phi_{p}\left(c, x_{n}\right)-\phi_{p}\left(u_{n}, x_{n}\right) \\
\phi_{p}\left(q, v_{n}\right) \leq \phi_{p}\left(q, y_{n}\right)-\phi_{p}\left(v_{n}, y_{n}\right) .
\end{array}
$$


From inequalities (3.2), (3.3) and (3.15), we have that

$$
\begin{aligned}
\phi_{p}\left(c, z_{n}\right) & \leq \alpha \phi_{p}\left(c, x_{n}\right)+(1-\alpha) \phi_{p}\left(c, \theta_{n}\right)-c_{p} w_{p}(\alpha)\left\|J_{p E_{1}} x_{n}-J_{p E_{1}} T \theta_{n}\right\|^{p} \\
& \leq \alpha \phi_{p}\left(c, x_{n}\right)+(1-\alpha)\left[\phi_{p}\left(c, u_{n}\right)-p \mu\left\langle A\left(\theta_{n}-c\right), e_{n}\right\rangle\right] \\
& \leq \phi_{p}\left(c, x_{n}\right)-(1-\alpha) \phi_{p}\left(u_{n}, x_{n}\right)-p \mu(1-\alpha)\left\langle A\left(\theta_{n}-c\right), e_{n}\right\rangle .
\end{aligned}
$$

Similarly,

$$
\phi_{p}\left(q, w_{n}\right) \leq \phi_{p}\left(q, y_{n}\right)-(1-\alpha) \phi_{p}\left(v_{n}, y_{n}\right)-p \mu(1-\alpha)\left\langle B\left(q-\delta_{n}\right), e_{n}\right\rangle .
$$

Using inequalities (3.17) and (3.18), the fact that $A c=B q$, inequality (3.10) and Step 2, we obtain that

$$
\phi_{p}\left(u_{n}, x_{n}\right)+\phi_{p}\left(v_{n}, y_{n}\right) \leq \frac{1}{(1-\alpha)}\left(\phi_{p}\left(c, x_{n}\right)-\phi_{p}\left(c, z_{n}\right)+\phi_{p}\left(q, y_{n}\right)-\phi_{p}\left(q, w_{n}\right)\right) .
$$

Thus,

$$
\lim _{n \rightarrow \infty} \phi_{p}\left(u_{n}, x_{n}\right)+\phi_{p}\left(v_{n}, y_{n}\right)=0 .
$$

By use of Lemma 2.6, we obtain that

$$
\lim _{n \rightarrow \infty}\left\|x_{n}-u_{n}\right\|=0 \quad \text { and } \quad \lim _{n \rightarrow \infty}\left\|y_{n}-v_{n}\right\|=0 .
$$

Step 4. Show that $\left(x^{*}, y^{*}\right) \in \Omega$ and $A x^{*}=B y^{*}$.

Since, for $p \geq 2$, each $p$-uniformly convex and uniformly smooth space is strictly convex, reflexive and smooth, we can apply the established result involving the functional $\phi$ instead of the functional, $\phi_{p}$. With this, the proof of Step 4 follows immediately by use of the same argument as in [18].

Remark 3.1. The condition on $\mu$ involves the norms, $\|A\|$ and $\|B\|$, respectively. This is not a drawback on implementing the algorithm because, for the computational purposes, one does not need to compute these norms. The norms can be replaced with two constants associated with the mappings, $A$ and $B$, which are easily obtained. To assert that a linear mapping $A$, is bounded, one has to show that $\|A x\| \leq K\|x\|, \forall x \in E$, and some constant $K>0$. This constant $K>0$, which is an upper bound for $\|A\|$, is generally easy to obtain (since it is not unique) for any bounded linear mapping. Similarly, to assert that a linear map $B$ is bounded, one has to show $\|B x\| \leq L\|x\|, \forall x \in E$, and some constant $L>0$. Again, this constant $L>0$ is easily obtained. It is easy to see from the proof of Theorem 3.1 that the condition

$$
0<\mu<\left[\frac{1}{\kappa_{p}\left(\|A\|^{\frac{p}{p-1}}+\|B\|^{\frac{p}{p-1}}\right)}\right]^{p-1},
$$

can be replaced with the condition

$$
0<\mu<\left[\frac{1}{\kappa_{p}\left(K^{\frac{p}{p-1}}+L^{\frac{p}{p-1}}\right)}\right]^{p-1},
$$

where $K$ and $L$ are easily obtained. 


\section{THE CONCLUSION}

The theorem of Monday [18] provides an algorithm, which is applicable in $L_{p}, l_{p}$ and the Sobolev spaces, $W_{p}^{m}(\Omega)$, where $1<p \leq 2$, for the problem studied because these spaces are 2-uniformly convex and uniformly smooth. The theorem is not applicable in $L_{p}, l_{p}$ and the Sobolev spaces, $W_{p}^{m}(\Omega)$, where $2<p<\infty$ because these spaces are not 2-uniformly convex. Our results provide an algorithm, which is applicable in these spaces which are $p$-uniformly convex and uniformly smooth. Consequently, our result complement the Monday's theorem and provide applicable algorithms in $L_{p}, l_{p}$ and the Sobolev spaces, $W_{p}^{m}(\Omega)$, for all $p$ such that $1<p<\infty$ for the problem.

\section{Acknowledgments}

This research was supported by the AfDB Research Grant Funds to the African University of Science and Technology, Abuja, Nigeria

\section{REFERENCES}

[1] H. Attouch, J. Bolte, P. Redont, A. Soubeyran, Alternating proximal algorithms for weakly coupled minimization problems. Applications to dynamical games and PDEs, J. Convex Anal. 15 (2008), 485-506.

[2] Y. Alber, Metric and generalized projection operators in Banach spaces: properties and applications, Theory and Applications of Nonlininear Operators of Accretive and Monotone Type (A. G. Kartsatos, Ed.), Marcel Dekker, pp. 15-50, New York, 1996.

[3] E. Blum, W. Oettli, From optimization and variational inequalities to equilibrium problems, Math. Stud. 63 (1994), 123-145.

[4] C. Byrne, A unified treatment of some iterative algorithms in signal processing and image reconstruction, Inverse Probl. 20 (2004), 103-120.

[5] A. Bnouhachem, A modified projection method for a common solution of a system of variational inequalities, a split equilibrium problem and a hierarchical fixed-point problem, Fixed Point Theory Appl. 2014 (2014), Article ID 22.

[6] S.S. Chang, L. Wang, Y. Zhao, Strongly convergent iterative methods for split equality variational inclusion problems in Banach spaces, Acta Math. Sci. 36 (2016), 1641-1650.

[7] Y. Censor, T. Bortfeld, B. Martin, A. Trofimov, A unified approach for inversion problems in intensitymodulated radiation therapy, Phys. Med. Biol. 51 (2006), 2353-2365.

[8] Y. Censor, A. Segal, The split common fixed point problem for directed operators, J. Convex Anal. 16 (2009), 587-600.

[9] C.E. Chidume, A. Adamu, L.C. Okereke, A Krasnoselskii-type algorithm for approximating solutions of variational inequality problems and convex feasibility problems, J. Nonlinear Var. Anal. 2 (2018), 203-218.

[10] C.E. Chidume, L.O. Chinwendu, A. Adamu, A hybrid algorithm for approximating solutions of a variational inequality problem and a convex feasibility problem, Adv. Nonlinear Var. Inequal. 21 (2018), 46-64.

[11] C.E. Chidume, M.O. Nnakwe, A new Halpern-type algorithm for a generalized mixed equilibrium problem and a countable family of generalized-J-nonexpansive mappings, with applications, Carpathian J. Math. 34 (2018), 191-198.

[12] C.E. Chidume, P. Ndambomve, A.U. Bello, The split equality fixed point problem for demi-contractive mappingpings, J. Nonlinear Anal. Optim 6 (2015), 61-69.

[13] C.E. Chidume, New geometric inequalities in real normed spaces with applications to iterative algorithms for solving split equality fixed point problems for quasi-phi-nonexpansive semigroups in real Banach spaces, (to appear).

[14] S. Kamimura, W. Takahashi, Strong convergence of a proximal-type algorithm in a Banach space, SIAM J. Optim. 13 (2002), 938-945. 
[15] J. Lindenstrauss, L. Tzafriri, Classical Banach Spaces II: Function Spaces, Ergebnisse Math. Grenzgebiete Bd., vol. 97. Springer, Berlin, 1979.

[16] Z. Ma, L. Wang, S.S. Chang, W. Duan, Convergence theorems for split equality mixed equilibrium problems with applications, Fixed Point Theory Appl. 2015 (2015), 31.

[17] A. Moudafi, Alternating CQ-algorithm for convex feasibility and split fixed-point problems, J. Nonlinear Convex Anal. 15 (2014), 809-818.

[18] M.O. Nnakwe, Solving split generalized mixed equality equilibrium problems and split equality fixed point problems for nonexpansive-type mappings, Carpathian J. Math. 36 (2020), 119-126.

[19] X. Qin, S.Y. Cho, Convergence analysis of a monotone projection algorithm in reflexive banach spaces, Acta Math. Sci. 37 (2017), 488-502.

[20] X. Qin, Y.J. Cho, S.M. Kang, Convergence theorems of common elements for equilibrium problems and fixed point problems in Banach spaces, J. Comput. Appl. Math. 225 (2019), 20-30.

[21] O.M. Romanus, U.V. Nnyaba, M.O. Nnakwe, Algorithms for a system of generalized mixed equilibrium problems and a countable family of some nonlinear multi-valued nonexpansive-type maps, Acta Math. Sci. 38 (2018), 1805-1820.

[22] W. Takahashi, K. Zembayashi, Strong and weak convergence theorems for equilibrium problems and relatively nonexpansive mappings in Banach spaces, Nonlinear Anal. 70 (2008), 45-57.

[23] H.K. Xu, Inequalities in Banach spaces with applications, Nonlinear Anal. 16 (1991), 1127-1138.

[24] J. Zhao, Solving split equality fixed-point problem of quasi-nonexpansive mappingpings without prior knowledge of operators norms, Optimization, 64 (2015), 2619-2630. 